\title{
雌雄异位和花部行为适应意义的研究进展
}

\author{
阮成江 姜国斌 \\ （大连民族学院民族地区生物资源与环境研究所, 辽宁大连 116600)
}

摘 要 雌雄异位和花部行为影响植物的传粉和交配格局。二者的适应意义一直是植物生殖生态学和进化生物 学研究的热点之一。该文概述了近年来雌雄异位和花部行为适应意义的研究进展, 包括对雌雄异位适应意义的最 早期、最传统的认识——避免自交和当前的主流观点——避免雌雄干扰; 花内性别干扰的形式及其例证; 花部行为 在避免雌雄功能干扰、避免自交、实现延迟自交、应对不良环境等方面的适应意义。讨论了使䧳雄异位程度减小的 锦葵科一些植物中的单体雄荵柱头运动可能涉及到的适应意义。针对目前在雌雄异位和花部行为适应意义研究 上存在的不足, 结合国际上研究二者适应意义的发展趋势正在从前期以描述性的研究为主转变到应用现代分子生 物学手段 (尤其是分子标记, 如 SSR、SNP、AFLP 等) 和传统方法相结合, 以及随着田间实验和控制条件能力的发展正 由经典的野外调查、观察为主过渡到包括花操纵在内的人工试验和野外试验相结合, 提出了今后研究中应注意的 问题。有必要借用多学科的手段, 以可作为研究䧳雄异位和花部行为的有着研究基础的植物为材料, 设计和操纵 不同的对比试验, 系统而深入地探讨雌雄异位和花部行为的适应意义。

关键词 雌雄异位 花部行为 单体雄荵柱头运动 适应意义

\section{ADAPTIVE SIGNIFICANCE OF HERKOGAMY AND FLORAL BEHAVIOUR}

\author{
RUAN Cheng-Jiang and JIANG Guo-Bin \\ ( Institute of Bio-Resources and Environment, Dalian Nationalities University, Dalian, Liaoning 116600, China)
}

\begin{abstract}
With their critical role in plant pollination and mating system, the adaptive significance of herkogamy and floral behavior has well been studied in plant reproductive biology and evolutionary ecology . Here we review recent progress in this field, especially discuss the adaptive significance of movement approach herkogamy in monadelphous tube in Malvaceae, which involves decrease of herkogamy resulting from the curvature of stigma lobe.
\end{abstract}

Herkogamy, the spatial separation of pollen presentation and pollen receipt within flowers, appears in many animal-pollinated plants. Due to its positive correlation with outcrossing rates in some species with mixed-mating systems, herkogamy has been previously considered as a mechanism to prevent self-pollination . However, increasing evidences suggest that herkogamy may act as a mechanism to reduce interference between the reproductive functions of female and male organs. Functional studies of heterostylous species indicate that sex-organ reciprocity serves to promote cross-pollen transfer between floral morphs and that enantiostyly and stigma-height dimorphism function in a similar manner.

Intra-floral sex interference often contains physical interference and pollen clogging. The former involves stamens obstructing the deposition of outcrossing pollen on stigmas and pistil reducing pollen export (an experimental evidence is given recently). The latter involves pollen discounting and ovule discounting, and its experimental evidence has been largely reported in many plants.

The behaviors of different floral parts ( style, stamen, pollen, corolla, etc. ) may have adaptive significance, including reducing intrafloral male-female interference, avoiding self-pollination, promoting delayed autonomous selfing, and responding to the harsh environment. Movement approach of herkogamy in monadelphous tube in Malvaceae, making the herkogamy decrease by the curvature of stigma lobe, is unusual and intriguing, and its adaptive significance may involves promoting outcrossing, facilitating delayed selfing if outcrossing fails, and interferences between two sexual organs.

We believe that the trends studying on the their functions are transiting from the research stage mainly depending on the description to comprehensive research phase combining modern experimental technology (e.g. the molecular markers of SSR, SNP, AFLP) and advanced apparatus multi-seasons with traditional method, 
and with the development of the capacity of field experiments and control conditions, transiting from traditional and classical observations to combining floral manipulation in the field experiments.

Key words Herkogamy, Floral behavior, Stigma movement in monadelphous tube, Adaptive significance

花, 被子植物的繁殖器官, 比所有其它类群有机 体的繁殖器官都表现出了更高的变异性(张大勇, 2004）。影响植物传粉和交配的花样繁多的花性状 ( Affre \& Thompson, 1998; Schemske \& Bradshaw, 1999; Peeters \& Totland, 1999; Takebayashi \& Delph, 2000; Bernardello et al., 2001; Singer \& Sazima, 2001; Caruso et al., 2003) 和灵活有趣的花部行为 (Li et al., 2001; Huang et al., 2002; Wang et al., 2004; Ruan et al., 2005a) 令许多传粉生物学家、生 态学家、进化生物学家和遗传学家着迷, 一直是植物 繁殖生态学和进化生物学研究的热点之一。花性状 可分为花设计 (Floral design) 和花展示 (Floral display) 两个层次 (张大勇, 2004)。花设计包括花的颜色 (Weiss，1991; Zufall \& Rausher，2004)、形状 (Dafni \& Kevan, 1997)、大小 ( Johnson et al ., 1995)、对称性 (Neal et al., 1998)、花粉数量和颜色 (Thien et al., 2000)、花粉胚珠比 ( Jacquemart, 2003 )、雌雄异位 (Pailler et al., 1998; Jesson \& Barrett, 2002)和䧳雄 异熟( Bertin \& Newman, 1993 )等; 花展示包括花在某 一时刻开放的数量和花在花序上的排列方式 (Roberston \& Macnair, 1995; Harder \& Barrett, 1995; Barrett, 2003; Mitchell et al., 2004）。花部行为包括 雌荵( 花柱)运动、雄荵运动和其它花部的运动。

Webb 和 Lloyd (1986) 认为, 雌雄异位既可以指 花内柱头和花药间的空间分离 (花内雌雄异位), 也 可以指同一植株上花与花表现出不一样的性别特征 和功能(花间雌雄异位)。作者采用 Barrett (2002a, $2002 b$ ) 及任明迅和张大勇 (2004) 的观点, 仅把花内 雌雄器官的空间分离视为真正的䧳雄异位。雌雄异 位在动物传粉的植物中非常普遍, 对其适应意义的 最早期、最传统的认识是避免自交，而当前的主流观 点则是避免雌雄干扰 ( Darwin，1876，1877; Webb \& Lloyd，1986）。近期国内外学者对雌雄异位的适应 意义进行了总结与介绍 (Barrett et al ., 2000; Barrett, 2002a，2002b; 任明迅和张大勇, 2004; 白伟宁 和张大勇, 2005）。但到目前为止, 尽管雌雄异位适 应意义的研究得到了迅速发展, 但还有许多方面不 为人知, 或没有定论, 如不同选择压力在某一雌雄异 位方式中的相对重要性及其出现先后(任明迅和张 大勇，2004）。
花部行为既包括外界环境诱导或访问者引发的 花部运动, 也包括遗传因素控制下的花部自身的主 动运动, 但不包括花药或花柱在访问者碰触下偶然 的被动的短暂晃动。自 $\operatorname{Darwin}(1862)$ 报道兰科植物 存在花部行为以来, 植物在长期进化过程中形成的 杂技般的、迷人的花部行为给研究花、传粉系统和繁 育系统的进化提供了激动人心的材料。但到目前为 止, 对某些奇妙的花部行为仍缺乏系统、深入的了 解, 给新植物繁殖生物学的研究提出了许多值得深 思和探讨的问题。例如, 花柱卷曲性的进化出现更 多地是为了避免自交还是避免雌雄功能的干扰呢? 锦葵科中的单体雄荵柱头运动如何协调和统一雌雄 干扰、促进异交和实现延迟自交等选择压力?

当然, 雌雄异位和花部运动常紧密相连。首先, 雌雄异位和花部行为的选择压力都涉及到避免雌雄 干扰; 其次, 花部性器官的运动大多发生在柱头探出 式雌雄异位中, 而动态式雌雄异位 (Movement herkogamy) 则是由花部 (雌芯 (柱头)、雄荵和其它花 部)的动态变化实现的; 最后, 锦葵科中一些植物中 的单体雄荵柱头运动将雌雄异位和花部行为更紧密 地结合在一起, 其适应意义可能涉及到避免雌雄干 扰、避免自交和实现延迟自交等多种选择压力。但 是, 花部行为和雌雄异位间也有一定区别, 如花部行 为的适应意义涉及到应对不良环境，包括应对不利 的自然环境(如气候条件)和应对不稳定的传粉者环 境，而适应不稳定传粉者环境的许多花部行为可实 现延迟自交, 这种自交一直滞后到异交机会丧失后 才发生, 提高了植物的结实率, 产生了繁殖保障效 应。

一个多世纪以来, 雌雄异位和花部行为的研究 得到了迅速发展, 这里仅就䧳雄异位和花部运动适 应意义的研究进展作一概述, 特别是初步探讨了通 过花部运动使雌雄异位程度降低的锦葵科单体雄荵 柱头运动的适应意义。

\section{1 䧳雄异位的适应意义}

雌雄异位有多种不同的形式, 任明迅和张大勇 (2004) 将其分为同型(柱头探出式雌雄异位、柱头缩 入式雌雄异位、动态式雌雄异位)和异型 (异长花柱、 镜像花柱、柱高二态)两大类。这里我们对雌雄异位 
适应意义从最早期、最传统的认识一一避免自交和 当前的主流观点一一避免雌雄干扰两方面进行介 绍:

\section{1 避免自交}

自 Darwin (1876) 以来, 花多样性 (包括䧳雄异 位) 的适应意义大多被认为是促进异交、避免自交的 机制 (Barrett, 2002b), 针对许多植物的研究为此提 供了有力证据。首先, 在许多自交亲和的物种中, 雌 雄异位影响母系的异交率 (Barrett, 2003), 且一些具 混合授粉机制物种的研究表明, 异交率与花药和柱 头间距离(雌雄异位) 成正比 (Holtsford \& Ellstrand, 1992; Belaoussoff \& Shore, 1995; Karron et al., 1997; Brunet \& Eckert, 1998; Motten \& Stone, 2000)。其 次, 柱头-花药间距离影响自花传粉 (Miyajima，2001; Elle \& Hare, 2002), 如一串红 (Salvia splendens) 中存 在雌雄异位和不存在雌雄异位的两种花在自花传粉 率上有较大不同 (Miyajima, 2001)。最后, 雌雄异位 影响近交衰退 (Stone \& Motten, 2002), 花 $\square$ 科的一年 生植物 Gilia achilleifolia 的近交衰退与雌雄异位间 存在显著正相关 (Takebayashi \& Delph, 2000)。但 是, 与前期对雌雄异位与异交率间存在明显相关性 的研究结果不同的是, Medrano 等(2005)最近的研究 表明, 石蒜科雌雄异位程度变异较大的植物 Narcissus longispathus 的异交率与雌雄异位间不存在正相 关, 这个结论被该研究解释为传粉者的不规律活动 造成的。

\section{2 避免䧳雄干扰}

由于许多具有雌雄异位的植物具有自交不亲和 性(Barrett, 2003), 一些学者最近提出, 雌雄异位的 主要功能是避免雌雄功能的干扰, 而不是仅仅作为 一种避免自交的机制 (Webb \& Lloyd, 1986; 任明迅 和张大勇, 2004)。性别干扰可以有不同形式 (Barrett, 2002b), 但最常见的花内雌雄干扰有两种重要 形式(Bertin \& Newman, 1993): 1)物理干扰。它指䧳 雄器官的位置或结构对雌雄功能产生的干扰, 包括 雄荵干扰柱头接受异交花粉和柱头干扰雄荵的花粉 输出两种情况。Parra-Tabla 和 Bullock (2005) 的研究 表明, 旋花科自交不亲和的 Ipomoea wolcottiana 的柱 头-花药间分离与柱头接受自身花粉数量间有显著 负相关, 柱头-花药间分离的增大降低了柱头上自花 花粉落置的数量, 并增加了柱头上异交花粉的落置 量, 避免了雄荵干扰柱头接受异交花粉。Fetscher (2001) 对玄参科 Mimulus aurantiacus 的动态雌雄异 位适应意义的研究结果支持了 Webb 和 Lloyd (1986)
的观点一一触敏柱头闭合可能有减小性别干扰的功 能。她发现, 与柱头闭合受抑制的植株相比, 授粉后 快速的柱头裂片闭合增大了异位程度并促进了花粉 在植株间的传播; 但值得注意的是, 她并没有发现这 种动态异位方式具有限制自交功能的证据; 2) 花粉 阻碍 (Pollen clogging)（Barrett，2002b)。对于自交不 亲和物种来说, 自花花粉落在柱头上可能会造成两 种后果: 胚珠折损 (Ovule discounting) 和花粉折损 (Pollen discounting)。支持花粉阻碍观点的例证较多 (详见 $\operatorname{Barrett}(2002 b)$ 及该文的参考文献)。因此, 避 免自交的花结构通过产生高质量的子代而从根本上 增加雌性适合度, 而减少雌雄繁殖器官干扰的花结 构则通过提高异交授粉的成功而在很大程度上促进 雄性适合度 (Barrett, 2003)。可见, 减少雌雄干扰可 能是一种独特的选择压力, 而不总为避免自交的副 产物 (Barrett, 2003)。

另外, 至少有 40 个靠动物传粉的科中存在雌雄 异位多态性 (Herkogamy polymorphism), 这不仅表明 雌雄异位不仅仅是避免自交的机制, 而且亦暗示它 是一种提高异交花粉传递精确性的机制 (Barrett, 2003; 任明迅和张大勇, 2004)。雌雄异位中的异长 花柱是一种提高异交花粉传递准确性的机制 (Darwin, 1877; Charlesworth \& Charlesworth, 1979; Lloyd \& Webb，1992）。近期对两个异型雌雄异位: 镜像花柱 (Jesson \& Barrett, 2002) 和柱高二态 (Thompson et $a l ., 2003)$ 的试验研究结果也得出了相似的结论。

到目前为止, 避免雌雄干扰假说日益被广泛接 受。白伟宁和张大勇(2005)用性别干扰理论解释了 各种花部性状(雌雄异熟、雌雄异位、单性花和自交 不亲和)的适应意义, 并强调通常被解释为避免自交 的花部机制实际上更大可能是为了避免性别干扰。 但是, 目前有关性别干扰假说的实验数据还有限, 更 多的是间接的推理和论证, 而且区别避免自交和避 免雌雄干扰是非常困难的 (白伟宁和张大勇, 2005)。因为自花授粉是避免自交假说的一个必要 条件, 但它也包含有干扰的一些形式。在许多有较 高异交率的、生理上自交不亲和的物种中, 许多也展 示出过去一直被解释为避免自交或“促进异交”的 “备份系统”( Back-up systems) (Barrett, 2002b)。因 此, 正如 Bertin 和 Newman( 1993) 指出的那样: 限制自 花授粉的适应性可能被选择, 因为它有避免自交和 (或者)减小性别干扰的优势。所以, 对雌雄异位适 应意义的研究将会在很长一段时间内仍是新植物繁 殖生物学领域的研究热点之一。 


\section{2 花部行为的适应意义}

影响传粉和交配格局的灵活多样的花部行为包 括: 1) 雌荵(花柱)运动, 如柱头裂片的闭合 (Fetscher \& Kohn, 1999; Yang et al., 2004)与反卷(Buttrose et al., 1977; Klips \& Snow, 1997; Ruan et al., 2004)、 花柱卷曲性( Li et al ., 2001; Zhang et al ., 2003)、花 柱向上 (Schlessman, 1986) 和向下弯曲 ( Faegri \& van der Pijl, 1979; Culley, 2002; Jesson \& Barrett, 2002; Verma et al.，2004) 及花柱弹射 (Kennedy，1999);2) 雄荵运动, 如雄荵向上弯曲 (Lyon, 1992) 和花丝伸长 ( Eckert \& Schaeffer, 1998; Traveset et al., 1998; Kalisz et al., 1999; Armbruster et al., 2002; Etcheverry et al., 2003); 3 ) 其它花部结构运动, 如花冠脱 落( Juncosa \& Webster，1989; Dole，1990; Donnelly et $a l ., 1998)$ 和闭合 (Ruan et al., 2004)、花粉滑动 (Wang et al., 2004) 与弹射 (Rathcke \& Real, 1993; Edwards et al., 2005) 及花粉块柄 (Caudicles)(van der Pijl \& Dodson, 1966) 和花柄的弯曲 ( Huang et al. , 2002)等。上述的花部行为的适应意义可归纳为以 下几种:

\section{1 避免雌雄功能干扰}

避免雌雄功能干扰可能是花部行为进化的一个 重要因子之一。雄全同株的多年生蔓生植物五叶爬 地虎 ( Passiflora incamata) 的雄花有两种花型: 一种花 型与其它雄全同株物种的雄花相似一一花柱萎缩 (Atrophy); 另一种花型的花柱与两性花相似,但花柱 一直处于直立状态而使柱头远离传粉者通道, 造成 柱头不能接受到花粉。然而, 五叶爬地虎两性花的 花柱向下弯曲使其柱头位于可能会与花粉携带者接 触的位置, 这可能在一定程度上避免了雄性器官的 位置对雌性功能的干扰 (Cindy, 2003)。美国凌霄 (Campsis radicans) 的柱头行为不是一种促进自花柱 头接受外来花粉的机制, 而是一种促进自花花粉散 发而提高雄性功能的机制( Yang et al., 2004)。自 交不亲和的御膳桔(Cornus canadensis)花粉的快速喷 射(Edwards et al.，2005)可能从两方面提高了花粉 的输出。首先, 当昆虫触动花后, 花粉喷射并粘到传 粉者身上, 随传粉者的运动而转移; 其次, 花粉喷射 后, 如果有风存在, 风传播花粉就会发生。具次级花 粉展现 (Secondary pollen presentation) 的竹芋科植物 中, 花开后花柱处于由帽状退化雄荵保持张力的一 种弯曲弹发状态, 花粉在花开放之前已脱落在刚好 位于柱头后花柱弯曲形成的浅凹处,一旦传粉者将
其头部插入花冠管, 压迫退化雄荵上的附属物 (板 机), 花柱弹发, 柱头与传粉者身体上的花粉接触, 而 花粉也同时被弹射到传粉者身上,这种花部行为既 促进了柱头接受花粉, 又有利于花粉的输出 (Kennedy，1999)。在玄参科的 Ourisia macrocarpa 中, 花开初期, 头状柱头伸出花冠管, 而雄荵位于花 冠管内, 花开大约 $5 \mathrm{~d}$ 后, 柱头授粉后向上弯曲, 花 冠管内的雄荵花丝伸长, 使得花药恰好位于花开初 期柱头所在位置, 从而减小了花粉接受和花粉输出 间的干扰 (Schlessman, 1986); 与 O. macrocarpa 相 似, 通过花部运动减少雌雄干扰的现象也发生在该 科的 Scrophularia nodosa 花中 (Faegri \& van der Pijl, 1979)。

我国学者 $\mathrm{Li}$ 等 $(2001 ， 2002)$ 在姜科植物中发现 了一种特殊的传粉机制一一花柱卷曲性。山姜属 (Alpinia) 植物种群有两种表型的个体: 花柱上举型 和花柱下垂型。柱头下垂型个体在刚开花时其柱头 向上反卷, 位于已开裂的花药上方, 到中午开始向下 运动, 下午则位于花药下方; 柱头上举型个体在开花 初期花柱向花冠内卷曲, 位于未开裂的花药和唇瓣 之间, 中午开始向上运动, 当柱头上举到花药上方 后, 花药开裂, 花粉开始散发 (张玲和李庆军, 2002; Zhang et al ., 2003)。张大勇(2004)及白伟宁和张大 勇 (2005) 倾向于认为花柱卷曲性是避免雌性功能干 扰雄性功能的一种行为学适应机制。首先, 柱头总 是远离昆虫拜访 (花粉扩散)通道这个现象, 可以看 成是避免接受自身花粉和避免干扰自身花粉的输 出。而且, 如果主要是为了避免自交, 那么雌性先熟 的花柱(上午已经接受了异株花粉)没有必要下午时 再向上举起, 除非后到达柱头的自身花粉, 相对于先 到达柱头的异株花粉, 具有较大的竞争优势。

\section{2 避免自交}

植物通过其花部的运动行为以避免自交或增加 异交率的现象已在多种植物中被报道(李庆军等, 2001), 如在玄参科的 O. macrocarpa 中, 由于雄荵和 花柱的运动使自交很少发生 (Schlessman, 1986)。 Verma 等(2004)的研究表明, 百合科植物 Eremurus himalaicus 的花柱运动起到了避免自交并促进异交 的作用。

\section{3 实现延迟自交}

延迟自交是一种被广泛报道的生殖适应性, 它 保证了植物在传粉者稀少情况下的种子生产, 但当 传粉者丰富时, 又允许异交优先发生。花发育过程 中产生延迟自交的花部运动包括: 1) 花晚期, 花柱分 
枝卷曲使柱头表面接触花药或接触落在非柱头区的 花粉, 这种花部行为发生在风铃草属的 Campanula spp. (Faegri \& van der Pijl, 1979), 堇菜属的Viola pubescens (Culley, 2002)，血皮草科的Wachendorfia brachyandra (Jesson \& Barrett, 2003), 锦葵科的野西 瓜苗( Hibiscus trionum) (Buttrose et al., 1977)、海滨 锦葵( Kosteletzkya virginica) (Ruan et al., 2004)和 $H$. laevis (Klips \& Snow，1997)等植物中;2)发生在山月 桂( Kalmia latifolia)(Rathcke \& Real, 1993) 和血根草 属的 Sanguinaria canadensis (Lyon, 1992)中的花后期 雄荵向上弯曲使花药与柱头相触; 3 ) 花晚期雄荵不 断向外露柱头伸长, 它发生在红蓸耧斗菜 (Aquilegia canadensis)(Eckert \& Schaefer, 1998)、短简倒挂金钟 ( Fuchsia magellanica) (Traveset et al., 1998)、三尖叶 猪屎豆 (Crotalaria micans) (Etcheverry et al ., 2003) 和 玄参科的 Collinsieae 簇植物 (Kalisz et al., 1999; Armbruster et al., 2002) 等植物中; 4) 黄花大苞姜 (Caulokaempferia coenobialis) 的花粉滑动 (Wang et $a l ., 2004) ; 5)$ 花晚期保持张力状态下弯曲花粉的弹 射 (Rathcke \& Real, 1993);6)花冠脱落过程中, 花冠 着生的雄荵的花药拂擦柱头, 这种行为发生在天蓝 色羽扇豆( Lupinus nanus)(Juncosa \& Webster, 1989)、 毛荵花( Verbascum thapsus) (Donnelly et al ., 1998)和 玄参科的 M. guttatus (Dole, 1990) 等植物中; 7 ) M. guttatus (Dole, 1990) 和蓝眼玛莉 (Collinsia verna) (Kalisz et al. ，1999)中的花柱运动与花冠脱落或海 滨锦葵 (Ruan et al., 2005a)中的柱头裂片运动与花 冠闭合同时发生。

\section{4 应对不良环境}

亚洲北温带地区的早春草本植物翁草 (Pulsatilla cernua) 的花柄在开花的 $6 \sim 10 \mathrm{~d}$ 内由直立到下垂 再到直立。Huang 等(2002) 认为这种花柄弯曲运动 对传粉者稀少的多雨生境下花粉生活力的保持可能 是重要的。位于北极的高山龙胆 (Gentiana algida) 在大雨前的花冠闭合可能是应对不良环境以提高生 殖适合度的策略, 因为在雷雨时不闭合花冠, 性器官 的生命力将会因雨淋而丧失 (Bynum \& Smith, 2001)。

但是, 正如很难区分雌雄异位适应意义中的避 免性别干扰和避免自交一样, 我们也很难区分某些 花部行为适应意义可能涉及到的避免自交和避免性 别干扰, 且二者常相互联系。玄参科的 $O$. macrocarpa 的雄荵和花柱运动 (Schlessman, 1986) 的适应 意义可能涉及到避免自交和避免雌雄干扰两方面。
百合科植物 $E$. himalaicus 的花柱运动起到了避免 自交并促进异交的作用 (Verma et al., 2004), 但其 花柱因运动而远离昆虫拜访的通道, 避免了雌荵对 花粉输出的影响, 这种花部行为有可能是一种促进 花粉散发而提高雄性适合度的机制。对于花柱卷曲 性, 尽管张大勇 (2004) 及白伟宁和张大勇 (2005) 更 倾向于认为它是一种行为学适应机制, 但花柱卷曲 性对于提高雄性和䧳性适合度方面的适应意义还需 要更多的实验和理论进行验证, 目前有的学者仍将 其归纳为避免自交或促进异交的行为机制 ( Li et $a l$. 2001, 2002; 李庆军等, 2001; 张玲和李庆军, 2002; Zhang et al., 2003)。延迟自交不仅无花粉折 损, 而且增加了种子产量 (Schoen \& Brown, 1991), 提 高了雄性和雌性适合度, 它是自交在某些情况下具 有适应意义的有利性状的例证, Kalisz 等(2004)的研 究为此提供了实例报道。同时, 延迟自交应对的是 不稳定的传粉者环境, 与上述的应对不良环境 (气候 条件, 如雨)有一定区别。鉴于以上分析, 本文将花 部行为的适应意义进行了上述归纳。另外, 有些花 部行为的适应意义可能未被归纳到上述类别中, 如 徐正尧等 (1997) 对桃儿七 ( Sinopodophyllum hexandrum) 的子房柄弯曲实现自花授粉现象的研究。

\section{3 单体雄芯柱头运动的适应意义}

柱头探出式雌雄异位 (Approach herkogamy) 是雌 雄异位中最常见的异位方式 (Barrett et al., 2000)。 在这种异位方式中, 花柱伸出花药之外, 传粉者进入 花时, 与其首先接触的是柱头 (Barrett et al., 2000), 如西番莲 (Passiflora caerulea) (任明迅和张大勇, 2004)。高高挑出的柱头还可能拦截到风中的花粉 (任明迅和张大勇, 2004); 花药-柱头间距离的增加 可以减少自花花粉落在自身柱头上的数量, 潜在地 增加异交授粉的比例 (Lloyd \& Schoen, 1992)。因 此, 柱头探出式雌雄异位既可以降低雌性功能(花粉 接受)与雄性功能(花粉散发)的相互干扰, 又可以避 免自花授粉 (Webb \& Lloyd, 1986; Barrett et al., 2000; 任明迅和张大勇, 2004)。然而, 如果性器官 分离太大, 传粉者在访花时可能只接触一套性器官 或只能以身体的不同部位接触它们, 将降低异交传 粉的准确度及植物的雄性和雌性适合度 (张大勇, 2004); 同时, 性器官分离太大也可能造成传粉者访 问期间对花柱的损害 (Parra-Tabla \& Bullock, 2005)。 Parra-Tabla 和 Bullock (2005) 的研究结果表明, 自然 选择支持有中间水平的柱头-花药间分离的 I. wol- 
cottiana 个体。

单体雄芯(花丝联合成包裹花柱的雄芯管, 形成 雌雄荵合生的柱状物) 是柱头探出式雌雄异位的一 种特殊形式, 主要出现在锦葵科植物中。在锦葵科 的单体雄荵中, 单体雄荵管紧抱花柱, 高低不等的花 药都位于通常五裂的、伸出雄荵管的柱头下方, 表现 出一种特殊的柱头探出式雌雄异位 (图 1a)。锦葵 科的单体雄芯有以下优点: 包裹花柱的雄芯管减小 了柱头、花药在花平面上的距离, 有利于花粉的接 受; 强度同时得到极大提高的雌雄芯可以承受传粉 者在花药与柱头上活动带来的压力 (任明迅和张大 勇, 2004）。从雌雄异位的角度来看, 锦葵科的单体 雄荵使得整个雄荵群(有多个不同高低的花药)位于 柱头的正下方, 自花花粉接触到柱头的可能性大大 降低; 但是, 如果性器官分离太大, 传粉者在访花时 可能只接触一套性器官或只能以身体的不同部位接 触它们的现象更易发生, 可能会降低异交传粉的准 确度及植物的雄性和䧳性适合度的可能性增大, 这 种雌雄干扰可归于花内干扰中的雄芯阻碍传粉者恰 当地在柱头落置而使肧珠未被受精。例如, 对于锦 葵科海滨锦葵来说, 花冠较大 (花冠直径为 $53.11 \pm$ $0.83 \mathrm{~mm}$ ), 很多传粉者采集花蜜和花粉时, 从侧面 直接进入花内, 又由于该物种的大多传粉者的体长 小于雄荵管 $(19.77 \pm 0.38 \mathrm{~mm}$ ) 及花柱长度 (雄芯管 和花柱伸出雄荵管长度之和, $23.40 \pm 0.37 \mathrm{~mm}$ ), 导 致仅与一套性器官 (雄芯) 接触。在江苏盐城滩涂海 滨锦葵种植地多年观察到的 12 种传粉者中, 仅胡蜂 总科的一个种 (体长在 $22.7 \mathrm{~mm}$ 左右) 进入花时首先 接触到的是柱头 (结果未发表)。在锦葵科单体雄萝 中, 不同物种柱头探出式雌雄异位程度变异较大, 有 些物种性器官分离较小 (如 H. asper、H. physaloides 和 H. sabdariff $a$ 等), 且在同一物种中, 雌雄异位程 度变异也较大, 如海滨锦葵的雌雄异位程度平均为 $3.31 \pm 0.04 \mathrm{~mm}(n=760)$, 范围在 $0.90 \sim 7.40 \mathrm{~mm}$ 之 间, 异位程度在群体内变异明显 $(C V=32.21 \%, S D$ $=1.07$ )(Ruan et al. 2005b)。因此, 分析锦葵科的 单体雄芯中性器官分离程度的适应意义对了解其进 化是非常必要的, 但到目前为止这方面的研究较少。 $\operatorname{Akpan}(2000$ ) 对具不同异位程度的木槿属 6 种植物 的研究表明, 开花前有无花药紧靠柱头 (异位程度) 直接影响物种的交配方式。柱头裂片有柄且开花前 没有花药紧靠柱头的物种为异交种, 如 H. acetosella 和 H. cannabinu。柱头裂片无柄且开花前有花药紧 靠柱头的物种为近交种, 如 H. asper、H. physa-
loides、H. sabdariffa 和 $H$. surattensis。这个例子暗示 单体雄芯的柱头探出式程度的适应意义可能涉及到 避免自交。

在各种不同的雌雄异位方式中, 除由花部行为 实现的动态式雌雄异位外, 花部性器官的运动大多 发生在柱头探出式雌雄异位中。锦葵科的植物中, 伸出单体雄荵管的柱头裂片在花期有两种表型: 一 种发生反卷运动, 可称为单体雄荵柱头运动, 已见报 道的物种有海滨锦葵( Ruan et al., 2005a)、野西瓜 苗 (Buttrose et al., 1977) 和 H. laevis (Klips \& Snow, 1997)等; 另一种不发生反卷运动, 如木槿 (H. syriacus)、树棉( Gossypium arboreum) 等。花部运动大多是 增加雌雄异位程度 (Fetscher, 2001), 但锦葵科的单 体雄芯柱头运动使雌雄异位程度减小 (有时减小为 零——柱头与自身花粉接触), 如以海滨锦葵为例, 说明单体雄芯柱头运动的过程, 海滨锦葵花在 $05: 00$ 前完全展开, 柱头裂片伸出单体雄芯管。柱头裂片 与单体雄荵管的长度方向几乎在同一条直线上(图 1a)。花开之后, 如果柱头一直未被授粉, 柱头裂片 将发生弯曲, 并持续(图 1b) 到柱头与自花花粉毗邻 (不接触) (图 1c) 或接触 (图 1d) 为止。如果刚刚开 放的花被授粉, 柱头裂片将不发生弯曲(图 1e)。柱 头裂片运动过程中, 一旦柱头接受到外来花粉, 运动 立即停止(图 1f)(Ruan et al., 2005a)。柱头裂片开 始运动时的柱头可授性约为 $64 \%$, 此时花粉生活力 在 $84.99 \%$ 左右; 柱头裂片运动停止时, 柱头可授性 $(90 \%)$ 和花粉生活力 ( $56.50 \%)$ 仍较高, 这为延迟自 交的发生提供了可能。

锦葵科单体雄荵柱头运动中, 仅未授粉的柱头 裂片发生反卷运动是独特而有趣的, 但对其适应意 义的解释也未形成统一观点。该种弯曲最初被认为 是一种巧妙的适应一一当异交机会存在时, 最小化 自花授粉, 如野西瓜苗的可逆花柱弯曲曾被认为是 一种首先支持异交, 但在异交失败时可灵活实现自 交的机制(Buttrose et al., 1977)。对同属植物 H. laevis, Stephens (1948) 将其花柱弯曲解释为一种有利 于异交的机制一一雄性阶段之后, 柱头裂片打开使 得柱头处于传粉者访问的路径上(雌性阶段开始); Blanchard (1976) 则认为它是一种实现自交的方法, 并引证所观察到的花晚期柱头因弯曲而能与自花花 粉相接触; Klips 和 Snow (1997) 的研究表明, 在 $H$. laevis 中, 部分种群通过花柱弯曲能实现自交, 但部 分种群由于柱头探出式雌雄异位程度太大而不能实 现延迟自交。对于存在上述的柱头裂片弯曲的海滨 

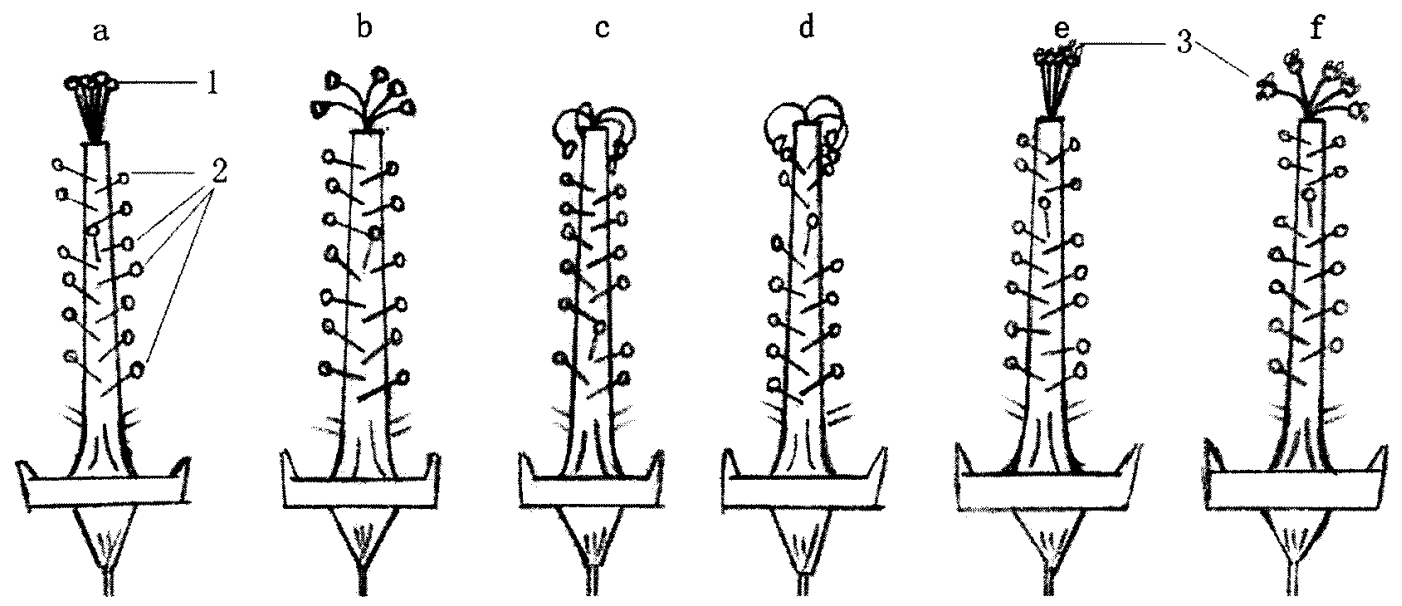

图 1 单花花期不同阶段的海滨锦葵花示意图 (Ruan et al., 2005a)

Fig. 1 Sketch map of flowers in Kosteletzkya virginica at different stages of flowering (Ruan et al., 2005a)

a: 刚刚开放的花, 柱头裂片与雄荵管长度方向几乎在同一直线上 Just opening flower, penta-lobed stigmas almost locate in the prolonged line of monadelphous tube $\mathrm{b}$ : 柱头裂片运动中未被授粉的花, 裂片运动持续了一段时间 Un-pollinated flowers being the curvature of stigma lobes, in which the curving movements last a certain time c: 柱头与自花花粉不接触(毗邻) Stigmas do not contact with (abut on) its own anthers late in flowering d: 柱头与 自花的花粉相触 Stigmas contact with its own anthers late in flowering e: 传粉发生在花开初期, 柱头裂片运动没有发生 Pollination occurring early in the anthesis（curvature of stigma lobes do not take place） f: 柱头裂片运动中被授粉的花,一旦柱头接受到花粉,裂片运动立即停止 Pollinated flower being the curvature of stigma lobes, in which the curving movements immediately stop once stigmas are pollinated 1 : 柱头裂片 Stigma lobes 2 : 花药 Anthers 3 : 花 粉粒 Pollen grains

锦葵, Ruan 等(2005a)指出, 在传粉者不足或缺乏时, 通过柱头裂片的反卷运动, 海滨锦葵可成功实现自 花授粉。海滨锦葵花中也存在部分由于柱头探出式 雌雄异位程度太大, 未授粉柱头裂片反卷运动停止 后柱头仍不能与自身花药接触的花, 但该类花在花 冠闭合作用下仍能成功实现自花授粉 (柱头裂片的 反卷运动是该类花实现延迟自交的前提) (Ruan et $a l ., 2005 \mathrm{a}$ )。作者据前期研究推测, 发生在锦葵科 海滨锦葵中的单体雄荵柱头运动的适应意义不仅仅 包括实现延迟自交,而且可能涉及到避免雌雄干扰 和促进异交两方面。首先, 海滨锦葵的单体雄萝是 一种特殊的柱头探出式雌雄异位, 因此具有避免雌 雄干扰的适应意义。柱头伸出单体雄荵管, 避免了 雄性对䧳性功能的物理干扰, 柱头首先接触传粉者 因而使干扰最小化; 柱头远离雄芯, 有利于花粉的输 出。可见,单体雄荵这种异位方式提高了自身花粉 的输出和异交花粉的获得, 从而减少了雄性功能和 雌性功能之间的冲突。其次, 海滨锦葵的单体雄范 柱头裂片反卷运动降低了雌雄异位程度, 可能会在 一定程度上避免上述的因性器官分离太大对雌雄功 能的干扰; 同时, 柱头裂片通过反卷靠近昆虫拜访的 通道, 有利于柱头接受花粉。而且, 在柱头回弯的前 半程有利于异交花粉的接触。因在回弯的前半程,
可接受花粉的柱头面位于访问者进入花的路径上, 便于异交花粉传递的准确性, 而在访问者退出花时 接触的是不能接受花粉的柱头一侧, 避免了自花授 粉的可能。再后, 海滨锦葵的柱头裂片在运动过程 中如果未接受到花粉, 将一直运动到与自身花粉接 触或毗邻 (降低或消除了雌雄异位程度), 实现了延 迟自交。这种基于单体雄荵柱头运动实现的延迟自 交, 利用的是不能再被利用的花粉, 在无花粉折损的 情况下, 保证了传粉者稀少或不足时的种子生产, 即 产生了繁殖保障效应, 提高植物雌性适合度的同时, 也提高了植物雄性适合度。这种情况下的延迟自交 可能是具有适应意义的有利性状。目前还没有确切 的证据去证明海滨锦葵中的柱头裂片反卷运动具有 几种适应意义和说明其中的哪一种更重要一些。

Barrett (2002a)认为三型花柱 (Tristyly) 种群中的 3 种交配类型也许代表了形态学适应昆虫传粉所能 达到的复杂性上限; 作者在此也认为锦葵科中海滨 锦葵的柱头裂片反卷运动与单体雄芯共同形成的形 态学适应昆虫传粉也达到了有花植物进化的完美结 合, 对其进行研究, 必将有助于进一步认识雌雄异位 和花部行为及其二者结合的适应意义。尽管柱头裂 片的反卷在很多植物中都有, 其延迟自交的现象也 在其它植物中有报道, 但海滨锦葵中的单体雄芯柱 
头裂片反卷运动有其独特的调节规律一一柱头裂片 运动是独自调节的, 即如果 5 个柱头中的 $1 \sim 4$ 个接 受到外来花粉, 那么仅接受花粉柱头的裂片运动立 即停止(授粉停止后的裂片保持停止时的状态, 不恢 复到直立状态一一卷是不可逆的), 其它未接受花 粉柱头裂片的反卷运动不受影响 (Ruan et al., 2005a)(目前这方面的定量实验工作正在进行)。而 对于有单体雄荵柱头运动报道的野西瓜苗, 单柱头 授以足量花粉对其它柱头裂片运动的抑制效果明 显, 且反卷是可逆的(授粉后的柱头裂片恢复到直立 状态)(Buttrose et al.，1977)。上述的海滨锦葵单体 雄荵 5 柱头裂片运动的独自调节规律可为研究花内 滞后自交提供研究材料,进而可能为从个体或花水 平上(目前未有实例报道)验证繁殖保障假说提供例 证。

\section{4 展 望}

在过去的一个多世纪, 许多关于雌雄异位适应 意义的有影响的研究都与避免自交有关, 这可能是 源于早期人们对该种花性状适应意义的认识。最 近, 关于雌雄异位适应意义一一避免雌雄干扰的重 新确认正日益被广泛重视, 这主要归功于最近理论 模型的发展和多领域的结合, 特别是进化生态学、遗 传学和系统学的结合。尽管花样繁多的雌雄异位一 直是生态学、进化生物学和遗传学研究的热点, 但雌 雄异位造成的交配模式和适合度的变化仍有许多不 为人知的方面, 且探索避免自交、避免雌雄功能干扰 和促进准确的异交传粉这些选择压力在各种植物小 小的花内如何相互妥协、怎样塑造出相应的花部综 合特征等问题,将仍然是植物繁殖生态学和植物进 化生物学中令人兴奋的研究领域(任明迅和张大勇, 2004)。

同样, 灵活多样的花部行为是植物长期适应其 生境所形成,对其适应意义的研究可为了解植物的 系统发育和进化提供资料, 并可为植物的引种、育种 提供科学依据。但到目前为止,一些花部行为的适 应意义仍不完全清楚, 尤其是单体雄荵柱头运动的 多样适应意义(可能会涉及到避免雌雄干扰、避免自 交和实现延迟自交)更令人着迷。例如, 尽管被认为 能提供繁殖保障的、由灵活有趣的花部运动所实现 的延迟自交在许多植物已见报道, 但验证它是否能 提供繁殖保障的例证较少，而多季节多种群内结合 多因子(自交方式及其所占比例、花粉折损、种子折 损、自交率和近交衰退等)对其进行验证的例证仅见
Kalisz 等(2004)及 Herlihy 和 Eckert(2002) 的报道。

最近高变异遗传标记在估计异交率上的应用 (如 RAPD、AFLP、SSR 等), 可能为前期以描述性的 研究为主的转变提供了一个很好的机会。特别是 SSR 标记在自交率估测、基因流定量研究、亲本分析 和传粉后的繁殖行为等研究中的应用, 可以实现对 雌雄异位和花部行为的直接作用和间接作用进行检 测, 特别是使检测雌雄功能的选择成为可能。同时, 随着田间实验和控制条件能力的发展, 通过包括花 操纵在内的人工试验, 可能使评价不同的性别干扰 形式及其潜在的生态和进化意义成为可能。因此, 在将来研究雌雄异位和花部行为的适应意义时, 我 们可将现代实验手段 (如 SSR、SNP 等分子标记) 和 现代操作技术与传统、经典的方法相结合, 利用多学 科的手段 (如生态学、遗传学、分子生物学、进化生物 学和比较生物学), 以可作为研究雌雄异位和花部行 为的有着研究基础的植物 (如 M. guttatus、姜科植 物、海滨锦葵等) 为材料, 设计和操纵不同的对比试 验 (设计的试验应能将避免自花授粉的功能与适应 意义从性状通过促进有效的花粉传播而增加交配机 会中区分开来), 系统而深入地探讨复杂的雌雄异位 和灵活有趣的花部行为的适应意义。具体研究内容 可涉及以下几方面: 1)验证现在少有实例报道的性 别干扰中的雄芯干扰柱头接受异交花粉; 2)测定雌 雄异位变异对花粉输出和自交率的影响, 操纵条件 下分析雌雄异位对交配系统及近交衰退的影响;3） 分析单体雄荵柱头运动的适应意义与适应性进化; 4)利用雌雄异位与雌雄异熟和雌雄同株等结合, 分 析花性状进化的选择压力一一异交和雌雄干扰的选 择; 5) 实验和比较多物种来评价这些物种共有的花 性状进化中雌雄干扰和自花授粉的相对重要性。

\section{参 考 文 献}

Affre L, Thompson JD (1998) . Floral traits variation in four Cyclamen (Primulaceae) species. Plant Systematics and Evolution, $212,279-293$.

Akpan GA (2000). Cytogenetic characteristics and the breeding system in six Hibiscus species. Theoretical and Applied Genetics, $100,315-318$.

Armbruster WS, Mulder CPH, Baldwin BG, Kalisz S, Wessa B, Nute H (2002). Comparative analysis of late floral development and mating-system evolution in tribe Collinsieae (Scrophulariaceae s. 1. ) . American Journal of Botany, 89, 37-49.

Bai WN (白伟宁), Zhang DY (张大勇) (2005). Sexual interference in cosexual plants and its evolutionary implications. Acta Phytoecologica Sinica (植物生态学报), 29,672-679. (in 
Chinese with English abstract)

Barrett SCH (2002a). The evolution of plant sexual diversity. $\mathrm{Na}$ ture Reviews Genetics, 3, 274-284.

Barrett SCH (2002b). Sexual interference of the floral kind. Heredity, 88, $154-159$.

Barrett SCH (2003) . Mating strategies in flowering plants: the outcrossing-selfing paradigm and beyond. Philosophical Transactions of the Royal Society of London, Series B, Biological Sciences, $358,991-1004$.

Barrett SCH, Jesson LK, Baker AM (2000). The evolution and function of stylar polymorphisms in flowering plants. Annals of Botany, 85, $253-265$.

Belaoussoff S, Shore JS (1995) . Floral correlates and fitness consequences of mating-system variation in Turnera ulmifolia. Evolution, 49, $545-556$.

Bernardello G, Anderson GJ, Stuessy TF, Crawford DJ (2001) . A survey of floral traits, breeding system, floral visitors, and pollination systems of the angiosperms of the Juan Fernandez Islands (Chile). Botanical Review, 67, 255 - 308 .

Bertin RI, Newman CM ( 1993 ). Dichogamy in angiosperms. Botanical Review, 59, $112-152$.

Blanchard OJ (1976). A Revision of Species Segregated from Hibiscus sect. Triomum (Medicus) de Candolle sensu lato (Malvaceae). $\mathrm{PhD}$ dissertation, Cornell University, Ithaca, New York, $63-71$.

Brunet J, Eckert CG (1998). Effects of floral morphology and display on outcrossing in blue columbine, Aquilegia caerulea (Ranunculaceae). Functional Ecology, 12, 596-606.

Buttrose MS, Grant WJR, Lott JNA (1977). Reversible curvature of style branches of Hibiscus trionum L., a pollination mechanism. Australian Journal of Botany, 25, 567 - 570 .

Bynum MR, Smith WK (2001). Floral movements in response to thunderstorms improve reproductive effort in the alpine species Gentiana algida (Gentianaceae). American Journal of Botany, $88,1088-1095$.

Caruso CM, Peterson SB, Ridley CE (2003). Natural selection on floral traits of Lobelia (Lobeliaceae) : spatial and temporal variation. American Journal of Botany, 90, 1333 - 1340 .

Charlesworth D, Charlesworth B (1979). A model for the evolution of distyly. American Naturalist, 114, $467-498$.

Cindy B (2003). Sex allocation and stylar movement in Passiflora incarnata. http://abstracts. co. allenpress. com/pweb/esa2003/ document $/$ ? ID $=26171$.

Culley TM (2002) . Reproductive biology and delayed selfing in $\mathrm{Vi}$ ola pubescens (Violaceae), an understory herb with chasmogamous and cleistogamous flowers. International Journal of Plant Sciences, 163, $113-122$.

Dafni A, Kevan PG (1997). Flower size and shape: implication in pollination. Israel Journal of Plant Science, 45, $201-212$.

Darwin C (1862). On the Various Contrivances by Which British and Foreign Orchids are Fertilized by Insects. John Murray, London,
$42-79$.

Darwin C (1876). The Effects of Cross and Self-Fertilization in the Vegetable Kingdom. John Murray, London, 471-482.

Darwin C (1877). The Different Forms of Flowers on Plants of the Same Species. John Murray, London, 123-135.

Dole JA (1990) . Role of corolla abscission in delayed self-pollination of Mimulus guttatus (Scrophulariaceae). American Journal of Botany, 77, 1505 - 1507 .

Donnelly SE, Lortie CJ, Aarssen LW (1998). Pollination in Verbascum thapsus (Scrophulariaceae): the advantage of being tall. American Journal of Botany, 85, 1618 - 1625.

Eckert CG, Schaefer A (1998). Does self-pollination provide reproductive assurance in Aquilegia canadensis (Ranunculaceae)? American Journal of Botany, 85, 919 - 924.

Edwards J, Whitaker D, Klionsky S, Laskowski MJ ( 2005 ). Botany: a record-breaking pollen catapult. Nature, 435, 164.

Elle E, Hare JD (2002) . Environmentally induced variation in floral traits affects the mating system in Datura wrightii. Functional Ecology, 16, 79 - 88 .

Etcheverry AV, Protomastro JJ, Westerkamp C (2003). Delayed autonomous self-pollination in the colonizer Crotalaria micans (Fabaceae: Papilionoideae): structural and functional aspects. Plant Systematics and Evolution, 239, 15-28.

Faegri K, van der Pijl L (1979). The Principles of Pollination Ecology 3rd edn. Pergamon Press, Oxford, 11-17.

Fetscher AE (2001). Resolution of male-female conflict in a hermaphroditic flower. Proceedings of the Royal Society of London, Series B, Biological Sciences, 268, 525-529.

Fetscher AE, Kohn JR (1999). Stigma behavior in Mimulus aurantiacus (Scrophulariaceae). American Journal of Botany, 86, $1130-1135$.

Harder LD, Barrett SCH (1995) . Mating cost of large floral displays in hermaphrodite plants. Nature, 373, 512-515.

Herlihy CR, Eckert CG (2002). Genetic cost of reproductive assurance in a self-fertilizing plant. Nature, 416, 320-323.

Holtsford TP, Ellstrand NC (1992). Genetic and environmental variation in floral traits affecting outcrossing rate in Clarkia tembloriensis (Onagraceae). Evolution, 46, 216-225.

Huang SQ, Takahashi Y, Dafni A (2002). Why does the flower stalk of Pulsatilla cernua (Ranunculaceae) bend during anthesis? American Journal of Botany, 89, 1599 - 1603.

Jacquemart AL (2003). Floral traits of Belgian Ericaceae species: are they good indicators to assess the breeding systems? Belgian Journal of Botany, 136, $154-164$.

Jesson LK, Barrett SCH (2002). Solving the puzzle of mirror-image flowers. Nature, 417, 707.

Jesson LK, Barrett SCH (2003). The comparative biology of mirrorimage flowers. International Journal of Plant Sciences, 164, S237 - 249.

Johnson SG, Delph LF, Elderkin CL (1995). The effect of petalsize manipulation on pollen removal, seed set and insect-visitor 
behavior in Campanula americana. Oecologia, 102, $174-179$. Juncosa AM, Webster BD (1989). Pollination in Lupinus nanus (Leguminosae) . American Journal of Botany, 76, 59-66.

Kalisz S, Vogler D, Fails B, Finer M, Shepard E, Herman T, Gonzales R (1999). The mechanism of delayed selfing in Collinsia verna (Scrophulariaceae). American Journal of Botany, 86, 1239 - 1247 .

Kalisz S, Vogler DW, Hanley KM (2004) . Context-dependent autonomous self-fertilization yields reproductive assurance and mixed mating. Nature, 430, $884-887$.

Karron JD, Jackson RT, Thumser NN, Schlicht SL (1997). Outcrossing rates of individual Mimulus ringens genets are correlated with anther-stigma separation. Heredity, 79, $365-370$.

Kennedy H (1999). Explosive secondary pollen presentation in family Marantaceae. Botanical Electronic News. Last Call for Botany BC \& Botwa 1999 Registrations [BEN \# 214]. http:// www. ou. edu/cas/botany-micro/ben/ben216. html. Cited 10 Mar.

Klips RA, Snow AA (1997). Delayed autonomous self-pollination in Hibiscus laevis (Malvaceae). American Journal of Botany, $84,48-53$.

Li Q, Kress WJ, Xu Z, Xia Y, Zhang L, Deng X, Gao J (2002). Mating system and stigmatic behaviour during flowering of Alpinia kwangsiensis (Zingiberaceae). Plant Systematics and Evolution, $232,123-132$.

Li Q, Xu Z, Kress WJ, Xia Y, Zhang L, Deng X, Gao J, Bai Z (2001). Flexible style that encourages outcrossing. Nature, 410,432 .

Li QJ (李庆军), Xu ZF (许再富), Xia YM (夏永梅), Zhang L (张玲), Deng XB (邓晓保), Gao JY (高江云) (2001). Study on the flexistyly pollination mechanism in Alpinia plants (Zingiberaceae). Acta Botanica Sinica (植物学报), 43, 364 - 369. (in Chinese with English abstract)

Lloyd DG, Schoen DJ (1992). Self- and cross-fertilization in plants. I. Functional dimensions. International Journal of Plant Sciences, $153,358-369$.

Lloyd DG, Webb CJ (1992). The selection of heterostyly. In: Barrett SCH ed. Evolution and Function of Heterostyly. Springer, Berlin, 179 - 208 .

Lyon DL (1992) . Bee pollination of facultatively xenogamous Sanguinaria canadensis L. Bulletin of the Torrey Botanical Club, $119,368-375$.

Medrano M, Herrera CM, Barrett SCH (2005). Herkogamy and mating patterns in the self-compatible daffodil Narcissus longispathus. Annals of Botany, 95, $1105-1111$.

Mitchell RJ, Karron JD, Holmquist KG, Bell JM (2004). The influence of Mimulus ringens floral display size on pollinator visitation patterns. Functional Ecology, 18, 116-121.

Miyajima D (2001). Floral variation and its effect on self-pollination in Salvia splendens. Journal of Horticultural Science \& Biotechnology, 76, $187-194$.
Motten AF, Stone JL (2000) . Heritability of stigma position and the effect of stigma-anther separation on outcrossing in a predominantly self-fertilizing weed, Datura stramonium (Solanaceae). American Journal of Botany, 87, 339 - 347.

Neal PR, Dafni A, Giurfa M (1998). Floral symmetry and its role in plant-pollinator systems; terminology, distribution and hypothesis. Annual Review of Ecology and Systematics, 29, 345 - 373.

Pailler T, Humeau L, Figier J, Thompson JD (1998). Reproductive trait variation in the functionally dioecious and morphologically heterostylous island endemic Chassalia corallioides (Rubiaceae) . Biological Journal of the Linnean Society, 64, 297 313 .

Parra-Tabla V, Bullock SH (2005). Ecological and selective effects of stigma-anther separation in the self-incompatible tropical tree Ipomoea wolcottiana (Convolvulaceae). Plant Systematics and Evolution, 252, $85-95$.

Peeters L, Totland O (1999). Wind to insect pollination ratios and floral traits in five alpine Salix species. Canadian Journal of Botany, 77, $556-563$.

Rathcke B, Real L (1993). Autogamy and inbreeding depression in Mountain Laurel, Kalmia latifolia (Ericaceae). American Journal of Botany, 80, $143-146$.

Ren MX (任明迅), Zhang DY (张大勇) (2004). Herkogamy. In: Zhang DY (张大勇) ed. Plant Life-History Evolution and Reproductive Ecology (植物生活史进化与繁殖生态). Science Press, Beijing, $302-320$. (in Chinese)

Roberston AW, Macnair MR (1995). The effects of floral display size on pollinator service to individual flowers of Myosotis and Mimulus. Oikos, 72, $913-925$.

Ruan CJ, Qin P, Han RM (2005a). Strategies of delayed self-pollination in Kosteletzkya virginica. Chinese Science Bulletin, 50, $94-96$

Ruan CJ, Qin P, Han RM (2005b) . Floral morphology and stigmaanther separation in gynomonoecious-gynodioecious Kosteletzkya virginica (Malvaceae). South African Journal of Botany, 71, $367-373$.

Ruan CJ, Qin P, He ZX (2004). Delayed autonomous selfing in Kosteletzkya virginica (Malvaceae). South African Journal of Botany, 70, 640-645.

Schemske DW, Bradshaw HD (1999). Pollinator preference and the evolution of floral traits in monkeyflowers (Mimulus). Proceedings of the National Academy of Sciences of the United States of America, 96, 11910 - 11915 .

Schlessman MA (1986). Floral protogyny, self-compatibility and the pollination of Ourisia macrocarpa (Scrophulariaceae). New Zealand Journal of Botany, 24, 651-656.

Schoen DJ, Brown AHD (1991). Whole- and part-flower self-pollination in Glycine clandestine and G. argyrea and the evolution of autogamy. Evolution, 45, 1665 - 1674.

Singer RB, Sazima M (2001). Flower morphology and pollination mechanism in three sympatric Goodyerinae orchids from south- 
eastern Brazil. Annals of Botany, 88, 989 - 997.

Stephens WC (1948). Kansas Wild Flowers. University of Kansas Press, Lawrence, Kansas, $41-56$.

Stone JL, Motten AF (2002) . Anther-stigma separation is associated with inbreeding depression in Datura stramonium, a predominantly self-fertilizing annual. Evolution, 56, 2187 - 2195.

Takebayashi N, Delph LF (2000). An association between a floral trait and inbreeding depression. Evolution, 54, 840-846.

Thien LB, Azuma H, Kawano S (2000). New perspective on the pollination biology of basal angiosperms. International Journal of Plant Sciences, 161, S225 - S235.

Thompson JD, Barrett SCH, Baker AM (2003) . Frequency-dependent variation in reproductive success in Narcissus: implications for the maintenance of stigma-height dimorphism. Proceedings of the Royal Society of London, Series B, Biological Sciences, 270, $949-953$.

Traveset A, Willson MF, Sabag C (1998). Effect of nectar-robbing birds on fruit set of Fuchsia magellanica in Tierra del Fuego: a disrupted mutualism. Functional Ecology, 12, 459 - 464.

van der Pijl L, Dodson CH (1966). Orchid Flowers: Their Pollination and Evolution. University of Miami Press, Coral Gables, 201 -207 .

Verma S, Magotra R, Koul AK (2004). Stylar movement avoids self-pollination and promotes cross-pollination in Eremurus himalaicus. Current Science, 87, 872-873.

Wang Y, Zhang D, Renner SS, Chen Z (2004). A new self-pollination mechanism. Nature, 431, 39-40.

Webb CJ, Lloyd DG (1986). The avoidance of interference be- tween the presentation of pollen and stigmas in angiosperms. II. Herkogamy. New Zealand Journal of Botany, 24, 163 - 178 .

Weiss MR (1991). Floral colour changes as cues for pollinators. Nature, 354, $227-229$.

$\mathrm{Xu}$ ZY (徐正尧), Ma SB (马绍宾), Hu CP (胡昌平), Yang CY (杨彩云), Hu ZH (胡志浩) (1997). The floral biology and its evolutionary significance of Sinopodophyllum hexandrum (Royle) Ying (Berberidaceae). Journal of Wuhan Botanical Research (武汉植物学研究) , 15, 223-227. (in Chinese with English abstract)

Yang SX, Yang CF, Zhang T, Wang QF (2004) . A mechanism facilitates pollination due to stigma behavior in Campsis radicans (Bignoniaceae). Acta Botanica Sinica (植物学报), 46,1071 1074.

Zhang DY (张大勇) (2004). Plant Life-history Evolution and Reproductive Ecology (植物生活史进化与繁殖生态). Science Press, Beijing, $100-113$. (in Chinese)

Zhang L (张玲), Li QJ (李庆军) (2002). Flexistyly and its evolutionary ecological significance. Acta Phytoecologica Sinica (植 物生态学报), 26, 385 - 390. (in Chinese with English abstract)

Zhang L, Li QJ, Deng XB, Ren PY, Gao JY (2003). Reproductive biology of Alpinia blepharocalyx (Zingiberaceae): another example of flexistyly. Plant Systematics and Evolution, 241, 67 -76 .

Zufall RA, Rausher MD (2004). Genetic changes associated with floral adaptation restrict future evolutionary potential. Nature, $428,847-850$. 